\title{
Erratum to: Yolky eggs prepare for metabolic acceleration
}

\author{
S. A. L. M. Kooijman
}

Published online: 11 June 2013

(C) Springer-Verlag Berlin Heidelberg 2013

Erratum to: J. Math. Biol. (2013) 66:795-805 DOI 10.1007/s00285-012-0597-7

After correction of an error in the code that was used to prepare the publication, it appears that yolky eggs do not prepare for metabolic acceleration. The error concerned the initial amount of egg mass, which now behaves consistently with theoretical predictions and roughly scales with body length to the power 4 among species.

Yolkiness of an egg is defined as the ratio of a largest and the smallest freshly laid egg, where the largest egg is produced by a well-fed mother and the smallest one has so little initial mass that it can invest just enough in maturation to reach birth, i.e. when assimilation is switched on. Metabolic acceleration is the factor with which the values for the maximum surface-area specific assimilation rate and the energy conductance of before birth, have to be multiplied to arrive at the values after metamorphosis. The maximum reserve capacity, i.e. the ratio of the maximum surfacearea specific assimilation rate and the energy conductance, is not affected by metabolic acceleration. For many species, this factor equals one, but for about one third of the now 240 species in the add_my_pet collection, the factor is larger than one. This collection includes representatives of most larger ( $>400$ species) animal phyla and all 13 chordate classes. Accelerating species include most species with a larval stage and a morphological metamorphosis in the juvenile period. Some accelerating species, such as the cephalopods, however, do not have such a distinct larval stage.

The online version of the original article can be found under doi:10.1007/s00285-012-0597-7.

S. A. L. M. Kooijman ( $\varangle)$

Department of Theoretical Biology, Vrije Universiteit, Amsterdam, The Netherlands

e-mail: bas@bio.vu.nl 
Yolkiness was thought to be proportional to acceleration. This idea was, however, based on an error in the code that finds patterns in the parameter values of species in the add_my_pet collection, which affects the unscaling of the scaled initial amount of reserve. Figure 1 presents the correct yolkiness as function of maximum structural length and of metabolic acceleration among species. No clear relationship exists between yolkiness and metabolic acceleration. Species that sport foetal development have been excluded from the figure, because foetusses receive resources during development. Insects also have been excluded because they skip the juvenile stage (larvae allocate to the reproduction buffer, which classifies them as adult in the context of DEB theory), accelerate after birth and switch to the imago stage if the relative size of reproduction buffer exceeds a threshold value.

If yolkiness exceeds the value 2, twinning, i.e. separation of cells in the 2-cell stage of embryo development, is theoretically possible. Both cells still have enough yolk to complete development, i.e. maturity can reach the threshold value at birth. Twinning is not only a remarkable biological phenomenon, but also an important technique to produce generically identical individuals for the study of phenotypic plasticity.

Fig. 1 The yolkiness, i.e. ratio of the maximum and minimum amount of initial reserve, $s_{E}^{0}$, as function of the maximum structural length, $L_{\infty}$ (top), and of the metabolic acceleration, i.e. the ratio of the lengths at metamorphosis and birth, $s_{\mathcal{M}}$ (bottom). The calculations are based on parameter estimates from the 240 species in the add_my_pet library. The open symbols (top) relate to species that sport metabolic acceleration $\left(s_{\mathcal{M}}>1\right)$. The colours refer to high-level taxa. The lines (top) refer to the threshold values $s_{E}^{0}=2,4$ and 8
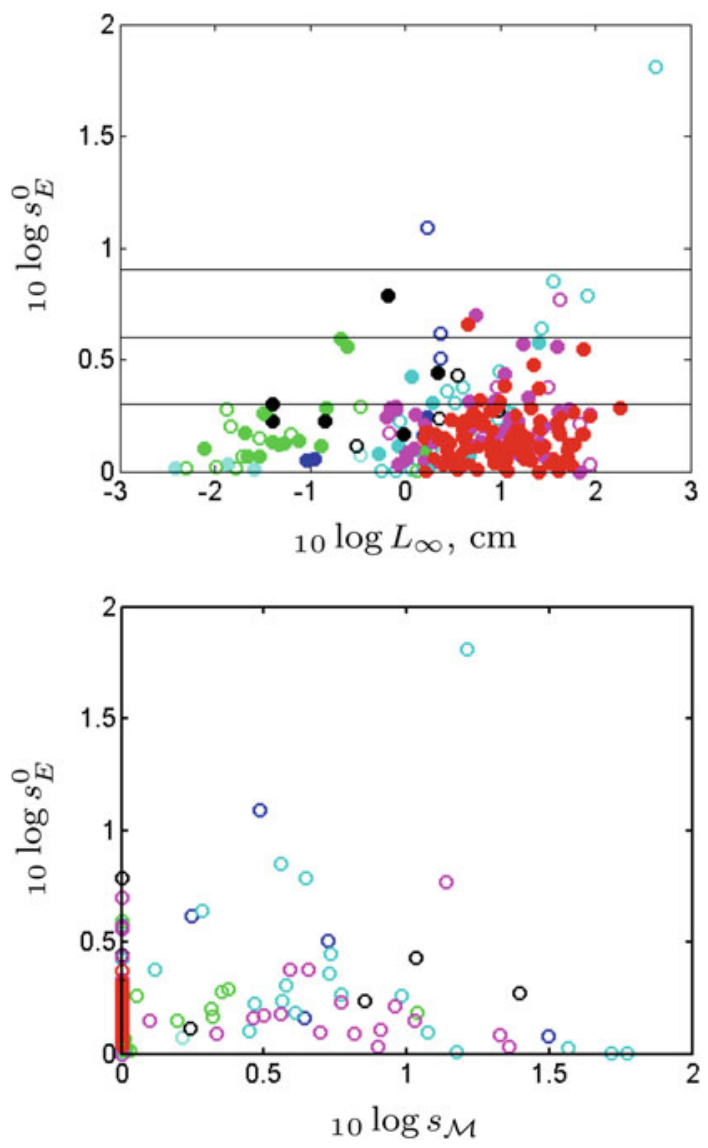
Figure 1 suggests that twinning can expected to be successful only for species that have a maximum structural length of at least $0.01 \mathrm{~cm}$. Separation of cells in the 4cell stage seems only possible in species that have a maximum structural length of at least $0.1 \mathrm{~cm}$. These results are consistent with the inter-specific co-variation rules for parameter values of DEB theory, which imply that maximum allocation to reproduction is expected to be proportional to maximum structural length to the power 3, initial egg mass to power 4 and a maximum reproduction rate to the power -1 . The figure also shows that quite a few large-bodied species exist where twinning is not possible.

Acknowledgments I like to thank Starrlight Augustine for spotting the error in the code that analyses patterns in parameter values of the add_my_pet collection and Bob Kooi for helpful comments. 